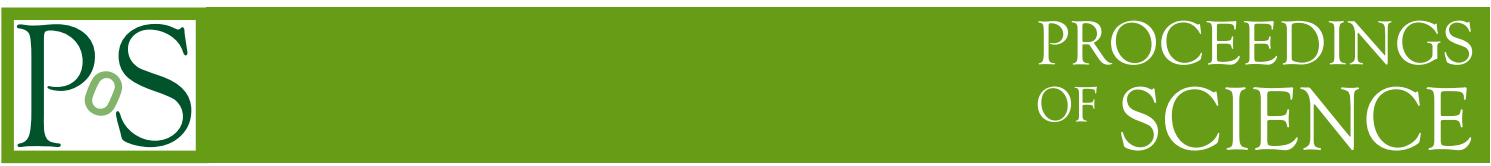

\title{
Heavy-ion collisions at ultra-relativistic energies
}

\author{
Ralf Averbeck*† \\ Research Division and ExtreMe Matter Institute EMMI, \\ GSI Helmholtzzentrum für Schwerionenforschung GmbH, \\ Planckstr. 1, 64291 Darmstadt, Germany \\ E-mail: R.Averbeck@gsi.de
}

\begin{abstract}
A brief overview is given on the experimental study of strongly-interacting matter at high energy density as produced in ultra-relativistic heavy-ion collisions, focusing on recent results from the Large Hadron Collider at CERN. Soft particle production is discussed in terms of collective effects, where similarities are observed between nucleus-nucleus collisions and $\mathrm{pp}$ and $\mathrm{p}-\mathrm{Pb}$ collisions, and the multiplicity-dependence of strange hadron yields in all collision systems. Hard probes of the produced Quark-Gluon Plasma (QGP) include jets and hadrons at high transverse momentum, where new measurements in $\mathrm{Pb}-\mathrm{Pb}$ collisions and, in particular, the analysis of $\mathrm{Xe}-$ Xe collisions can provide further details on radiative parton energy loss in the QGP. New results on the production of open heavy-flavor hadrons provide further insight into the transport of heavy charm and beauty quarks in the dense medium and their interactions with it. Finally, measurements on the production of quarkonia give evidence for deconfinement in $\mathrm{Pb}-\mathrm{Pb}$ collisions at the LHC and could help to determine the initial temperature of the produced QGP.
\end{abstract}

The 39th International Conference on High Energy Physics (ICHEP2018)

4-11 July, 2018

Seoul, Korea

\footnotetext{
* Speaker.

${ }^{\dagger}$ Discussions with the participants of the heavy-ion parallel session are gratefully acknowledged.
} 


\section{Introduction}

With collisions of heavy nuclei at ultra-relativistic energies the many-body physics of Quantum Chromo-Dynamics (QCD) at high energy density $\varepsilon$ can be investigated (see [1] for a recent review). For $\varepsilon \approx 1 \mathrm{GeV} / \mathrm{fm}^{3}$, lattice QCD calculations predict a phase transition from matter composed of hadrons to a Quark-Gluon Plasma (QGP), in which the quarks and gluons are no longer confined but can move over distances much larger that the size of a nucleon (see [2] for an early review). It has been shown that at zero baryochemical potential $\mu_{B}$, i.e. at vanishing net-baryon density, the deconfinement phase transition is linked to the restoration of chiral symmetry and that it is of crossover type [3] with a continuous, smooth change of thermodynamic quantities close to the pseudo-critical temperature $T_{c} \approx 156 \mathrm{MeV}$ [4]. This region of the QCD phase diagram is explored via heavy-ion collisions at ultra-relativistic energies.

First evidence for the existence of the QGP was obtained from experiments at the CERN SPS with collisions of $\mathrm{Pb}$ nuclei at a centre-of-mass energy per nucleon pair of $\sqrt{s_{\mathrm{NN}}}=17.2 \mathrm{GeV}$. Decisive proof for the production of the QGP came from RHIC at BNL with measurements of $\mathrm{Au}-\mathrm{Au}$ collisions up to $\sqrt{s_{\mathrm{NN}}}=200 \mathrm{GeV}$. The results from RHIC characterized the QGP as a nearly ideal, strongly interacting fluid in contrast to a weakly interacting gas of quarks and gluons. Measurements at the CERN LHC at $\sqrt{s_{\mathrm{NN}}}=2.76$ and $5.02 \mathrm{TeV}$ confirmed the findings from RHIC and extended the characterization of the QGP properties into unexplored territory in phase space, with much increased precision and new, previously unavailable probes.

A heavy-ion collision is a complex, highly dynamical process. Schematically, six stages can be identified: 1) initial parton scattering processes taking place while the two Lorentz-contracted nuclei pass through each other, 2) thermalization and formation of the deconfined QGP, 3) hydrodynamic expansion and cooling of the QGP, 4) hadronization from a QGP to a hadron gas, 5) chemical freeze-out when inelastic collisions cease and hadron yields are fixed, and 6) kinetic freeze-out when elastic collisions cease and spectra as well as correlations are fixed. The characterization of properties of the QGP in stage 3) is challenging and requires additional input from model comparisons. Furthermore, it is crucial to establish an experimental baseline reflecting the case in which no deconfined medium is formed. In the traditional approach, this role is taken by pp collisions, and p-A collisions are investigated to quantify cold nuclear matter effects due to the presence of a nucleus in the initial state, e.g. nuclear shadowing of the parton distribution functions leading to a reduced luminosity for hard parton scattering processes with respect to pp collisions.

Recently, the observation of correlations in high-multiplicity pp and p-A collisions, reminiscent of those correlations that have been shown to be due to truly collective effects in heavy-ion collisions, have triggered intensive discussion as to whether the underlying physics is the same in heavy-ion collisions and high-multiplicity collisions of small systems. This debate boils down to the question how small a droplet of QGP can be and, depending on the answer, the heavy-ion physics paradigm that pp collisions constitute a pure non-QGP reference might shift. At the same time, the performance of pp event generators used conventionally in high-energy particle physics has to be questioned for high-multiplicity collisions, where current implementations have difficulties describing the data. Deeper insight on the physics of high energy density QCD has been obtained from recent measurements in nucleus-nucleus and in small collision systems at RHIC and, in particular, at the LHC as will be discussed based on selected results in the following. 


\section{Soft probes}

Even at LHC energies more than $95 \%$ of all particles are produced with transverse momenta $p_{\mathrm{T}}$ below $2 \mathrm{GeV} / c$ in pp and heavy-ion collisions. Azimuthal anisotropies $v_{n}$, which are the Fourier coefficients of the azimuthal distributions of produced particles with respect to the orientations of the corresponding symmetry planes, are among the most important observables that probe the soft physics in the non-perturbative QCD regime.

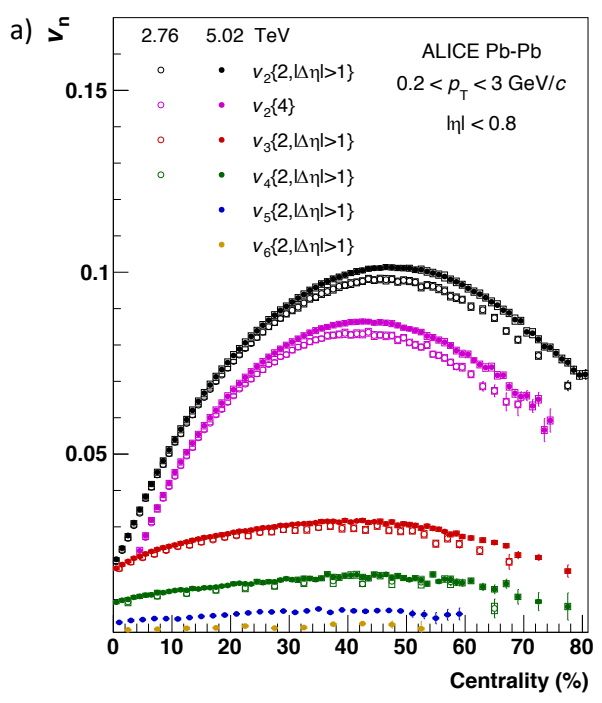

Figure 1: a) Flow coefficients $v_{n}$ of charged particles versus centrality in $\mathrm{Pb}-\mathrm{Pb}$ collisions at $\sqrt{s_{\mathrm{NN}}}=2.76$ and $5.02 \mathrm{TeV}$ (from [5]). b) $v_{2}$ versus multiplicity in $\mathrm{pp}, \mathrm{p}-\mathrm{Pb}, \mathrm{Xe}-\mathrm{Xe}$, and $\mathrm{Pb}-\mathrm{Pb}$ collisions at $\sqrt{s_{\mathrm{NN}}}=13,5.02,5.44$, and $5.02 \mathrm{TeV}$, respectively. c) Protons and strange hadron yields relative to pion yields in pp collisions at $\sqrt{s}=7$ and $13 \mathrm{TeV}, \mathrm{p}-\mathrm{Pb}$ and $\mathrm{Pb}-\mathrm{Pb}$ collisions at $\sqrt{s_{\mathrm{NN}}}=5.02 \mathrm{TeV}$, and $\mathrm{Xe}-\mathrm{Xe}$ collisions at $\sqrt{s_{\mathrm{NN}}}=5.44 \mathrm{TeV}[9]$.
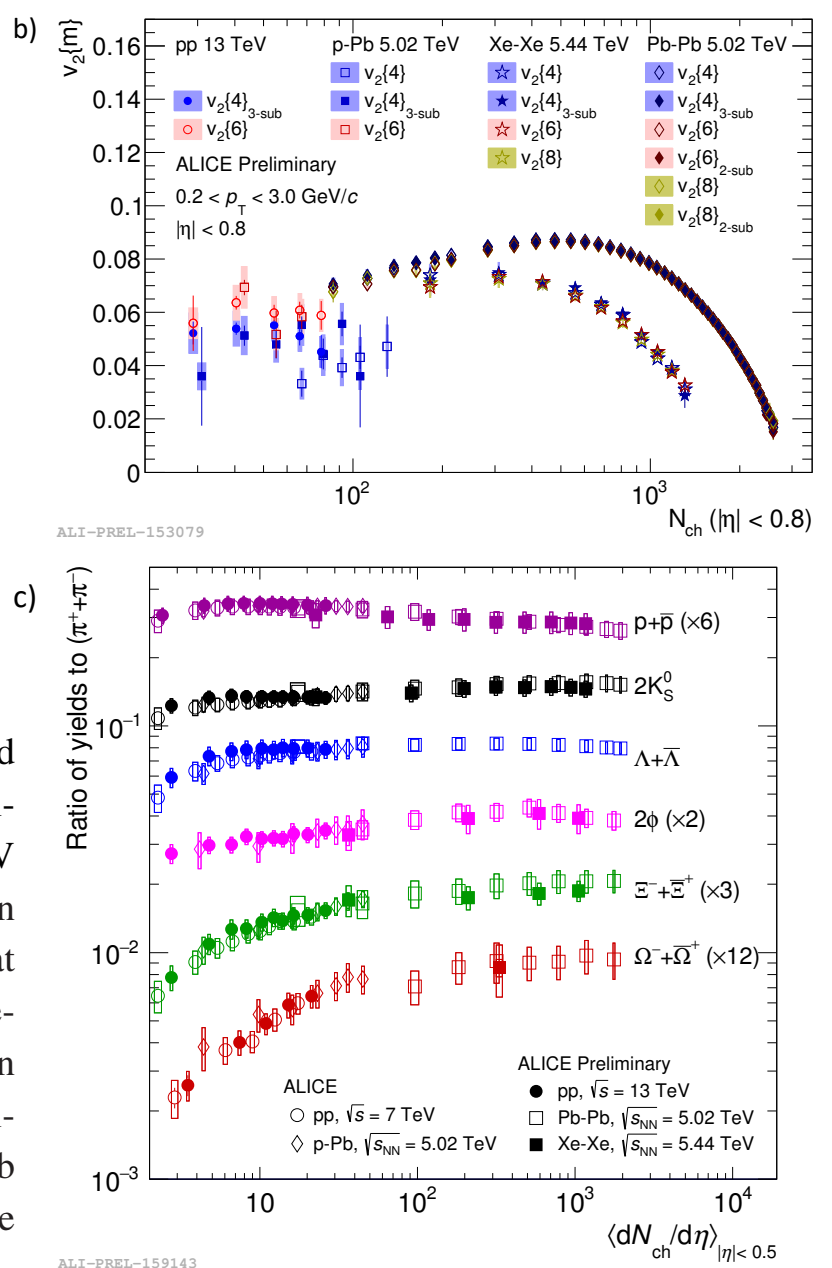

Fig. 1a shows the $p_{\mathrm{T}}$-integrated $v_{2}$ and higher coefficients up to $v_{6}$ as a function of centrality in $\mathrm{Pb}-\mathrm{Pb}$ collisions [5]. In peripheral and semi-central collisions, $v_{2}$ is much larger than the higher harmonics while this is not the case for central collisions. Since the higher harmonics are driven by fluctuations of the initial energy density profile, this observation demonstrates that in central collisions fluctuations have an important impact on azimuthal anisotropies while towards more peripheral collisions the initial geometry prevails.

Detailed measurements of $v_{2}$ have been performed using multi-particle cumulants in order to suppress non-flow contributions also in smaller collision systems and as a function of the multiplicity of produced charged particles. The data compilation in Fig. $1 \mathrm{~b}$ shows $v_{2}$ values in highmultiplicity $\mathrm{pp}$ and $\mathrm{p}-\mathrm{Pb}$ collisions similar to those measured in peripheral nucleus-nucleus col- 
lisions at the LHC, suggesting collective behavior even in the smallest collision system. Also in other correlation measurements in high-multiplicity $\mathrm{pp}$ and $\mathrm{p}-\mathrm{Pb}$ collisions features are observed that are similar to those interpreted as due to collective flow in nucleus-nucleus collisions $[6,7,8]$.

A smooth transition from small to large collision systems is also observed elsewhere. Yields of protons and hadrons carrying strange quarks are normalized to pion yields and shown as a function of the charged-particle multiplicity in pp, $\mathrm{p}-\mathrm{Pb}, \mathrm{Xe}-\mathrm{Xe}$, and $\mathrm{Pb}-\mathrm{Pb}$ collisions in Fig. 1c [9]. The smooth evolution of particle production with multiplicity might point to a common origin in all collision systems. The smooth increase of strangeness production towards its plateau values starts already in pp collisions. Conventional pp event generators are challenged by these finding [10]. It will be crucial to study the role of self interaction / color reconnection in QCD in the context of (multi-)particle production in more detail in the future.

\section{Hard probes}

Further information on the properties of the Quark-Gluon Plasma can be obtained via hard probes. Partonic scattering processes with large momentum transfer in the early stages of the collision produce high-momentum or heavy partons which propagate through the deconfined medium and interact with its color charges thus loosing energy via gluon radiation or collisions. Parton energy loss leads to the phenomenon of jet quenching which has been investigated via many observables including the suppression of jet and leading hadron yields at high $p_{\mathrm{T}}$.

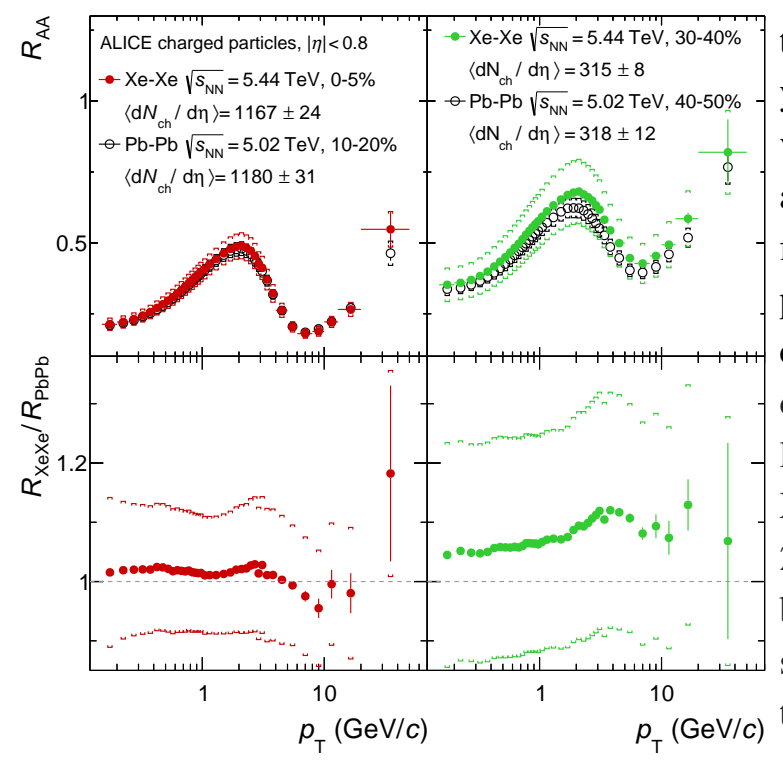

This suppression is usually quantified via the nuclear modification factor $R_{\mathrm{AA}}$, i.e. the yield measured in nucleus-nucleus collisions divided by the product of the pp cross section and the nuclear overlap integral, which quantifies how many nucleon-nucleon collisions take place on average in an AA collision of a given centrality. In Fig. 2 nuclear modification factors of charged particles [11] measured in $\mathrm{Pb}-\mathrm{Pb}$ collisions are compared with those from a six-hour $\mathrm{Xe}-\mathrm{Xe}$ run, which took place at the the LHC in 2017. In both systems a maximum suppression by a factor of $\approx 5$ is observed in central collisions for $5<p_{\mathrm{T}}<10 \mathrm{GeV} / c$. $R_{\mathrm{AA}}$ increases towards peripheral collisions and scales approximately with the charged-particle multiplicity, i.e. Figure 2: $R_{\mathrm{AA}}$ of charged particles as a function of $p_{\mathrm{T}}$ in central and semi-central Xe-Xe collisions compared with $R_{\mathrm{AA}}$ in $\mathrm{Pb}-\mathrm{Pb}$ collisions with similar charged-particle multiplicity $\left\langle d N_{\mathrm{ch}} / d \eta\right\rangle$ (from [11]).

$R_{\mathrm{AA}}\left(p_{\mathrm{T}}\right)$ is similar in both systems for those centrality selections which result in similar volumes but different shapes of the fireball. This opens up new venues to study e.g. the path-length dependence of partonic energy loss.

In the small $\mathrm{pp}$ and $\mathrm{p}-\mathrm{Pb}$ systems no evidence for jet quenching is observed $[12,13]$ which, seemingly, is at odds with the apparent collectivity in high-multiplicity $\mathrm{pp}$ and $\mathrm{p}-\mathrm{Pb}$ collisions. 
However, it has been shown [14] that only a few, elastic scatterings are necessary to generate a positive $v_{2}$, keeping the energy loss small. More detailed studies on the event-activity dependence of energy loss are clearly needed in small systems.

A comparison of $R_{\mathrm{AA}}\left(p_{\mathrm{T}}\right)$ of hadrons [13] and high- $p_{\mathrm{T}}$ jets [15] is shown in Fig. 3. While for hadrons $R_{\mathrm{AA}}$ approaches unity at high $p_{\mathrm{T}}$, consistent with the expected $\log E$ dependence of partonic energy loss, jets are suppressed $\left(R_{\mathrm{AA}}<1\right)$ up to transverse momenta as large as $1 \mathrm{TeV} / c$, suggesting an increase of the energy loss with increasing energy. With the jet energy the number of energy-loosing partons in the jet grows, which might lead to a growth of the jet energy loss with the jet energy. This first glimpse of jets as scale-dependent probes could open up the $p_{\mathrm{T}}$ dependence of jet modifications as a new field of study.
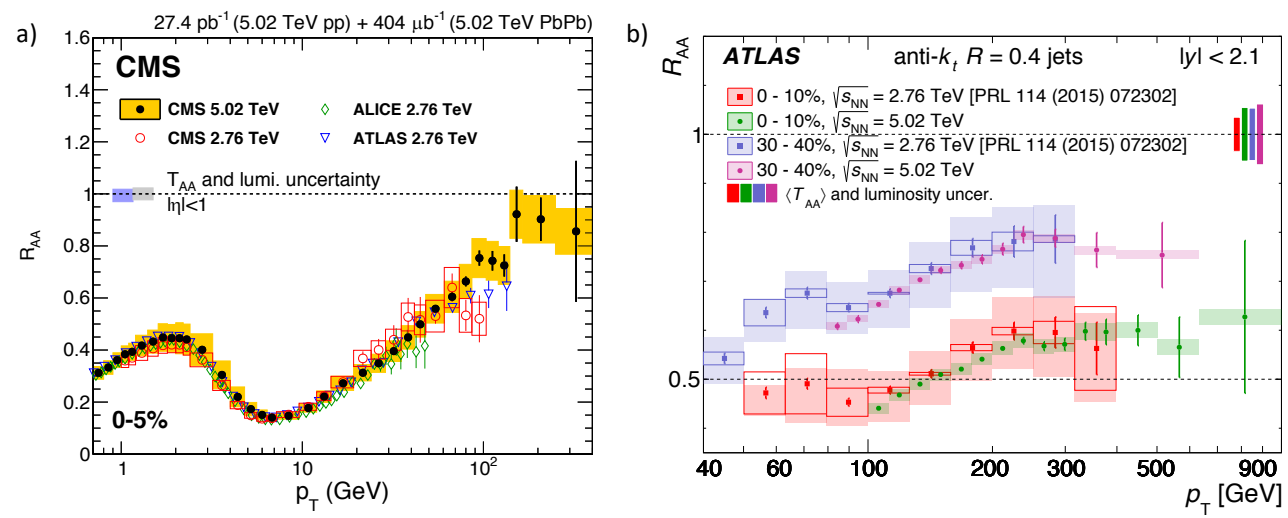

Figure 3: a) $R_{\mathrm{AA}}$ of charged particles as a function of $p_{\mathrm{T}}$ in central $\mathrm{Pb}-\mathrm{Pb}$ collisions at $\sqrt{s_{\mathrm{NN}}}=2.76$ and 5.02 TeV (from [13]). b) Jet $R_{\mathrm{AA}}$ as a function of $p_{\mathrm{T}}$ in central and semi-central $\mathrm{Pb}-\mathrm{Pb}$ collisions at $\sqrt{s_{\mathrm{NN}}}=2.76$ and $5.02 \mathrm{TeV}$ (from [15]).
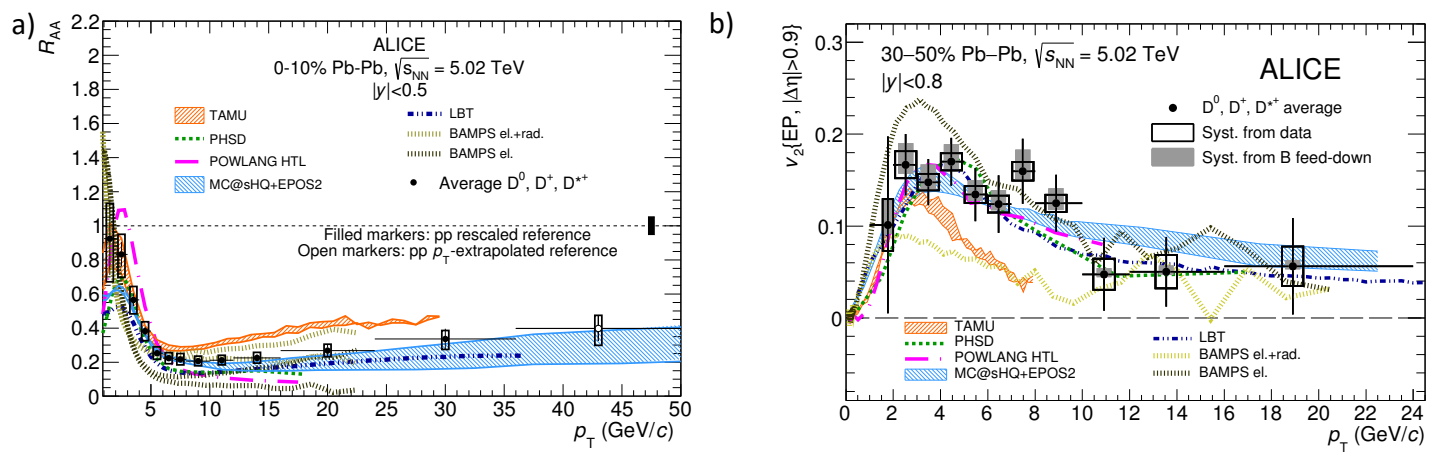

Figure 4: $R_{\mathrm{AA}}$ (a) and $v_{2}$ (b) of non-strange $\mathrm{D}$ mesons as a function of $p_{\mathrm{T}}$ in central and semi-central $\mathrm{Pb}-\mathrm{Pb}$ collisions, respectively, at $\sqrt{s_{\mathrm{NN}}}=5.02 \mathrm{TeV}$ (from [16]).

Due to their large masses, heavy quarks, i.e. charm and beauty, are produced almost exclusively in initial hard partonic scattering processes, even down to zero $p_{\mathrm{T}}$ which distinguishes heavy-flavor production from jet production. The formation time of heavy quark-antiquark pairs is smaller than the formation time of the Quark-Gluon Plasma, and the quark flavor is preserved in strong interactions in the medium. Therefore, the heavy quarks keep their identity while they propagate through the dense medium, interact with it and, potentially, approach thermalization. These unique probes experience and, to some extent, participate in the full medium evolution. 
Charm quarks lose energy and participate in the collective flow in $\mathrm{Pb}-\mathrm{Pb}$ collisions at the LHC as demonstrated in Fig. 4, which shows the average nuclear modification factor $R_{\mathrm{AA}}$ and elliptic flow coefficient $v_{2}$ of non-strange $\mathrm{D}$ mesons as a function of $p_{\mathrm{T}}$ in central and semi-central $\mathrm{Pb}$ $\mathrm{Pb}$ collisions, respectively [16]. A strong suppression of the yield at intermediate and high $p_{\mathrm{T}}$ as well as a positive $v_{2}$ are observed. The data are compared with results from hydrodynamic and microscopic transport calculations. The requirement to describe simultaneously $R_{\mathrm{AA}}$ and $v_{2}$ starts to constrain the model parameters, and efforts are ongoing [17] to extract transport properties of the dense medium for the comparison of data and model predictions.

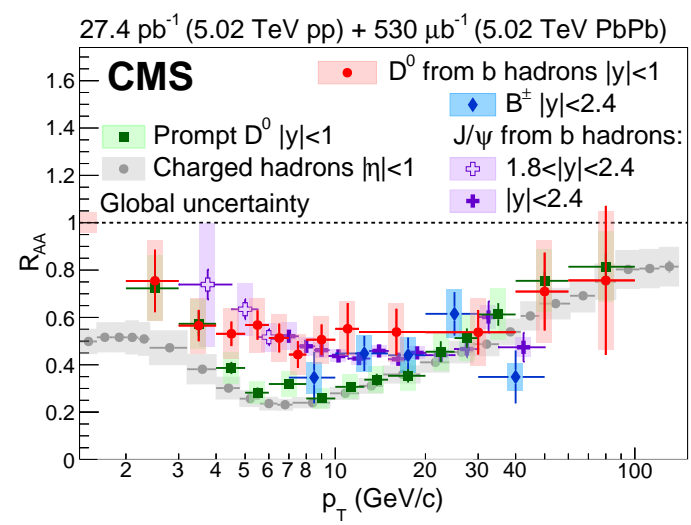

Figure 5: Nuclear modification factor $R_{\mathrm{AA}}$ of neutral $D$ mesons and $J / \psi$ from $b$ hadron decays, charged B mesons, prompt neutral $\mathrm{D}$ mesons, and charged hadrons as a function of $p_{\mathrm{T}}$ in $\mathrm{Pb}-\mathrm{Pb}$ collisions at $\sqrt{s_{\mathrm{NN}}}=5.02 \mathrm{TeV}$ (from [18]).
The measurement of $R_{\mathrm{AA}}$ of hadrons carrying beauty quarks as shown in Fig. 5 for $\mathrm{Pb}-\mathrm{Pb}$ collisions at $\sqrt{s_{\mathrm{NN}}}=5.02 \mathrm{TeV}$ [18] provides insight into the mass dependence of partonic energy loss. Nuclear modification factors of charged B mesons as well as neutral $\mathrm{D}$ mesons and $\mathrm{J} / \psi$ from b-hadron decays are compared with $R_{\mathrm{AA}}$ of prompt neutral D mesons and charged hadrons. The yields of charm mesons tend to be less suppressed than beauty mesons and their decay products, even though for the latter the decay kinematics has to be considered when comparing with prompt D mesons. The observed mass ordering between charm and beauty hadrons can be explained by the dead-cone effect, i.e. a decrease of the radiative energy loss with increasing parton mass [19].

New data on $\Lambda_{\mathrm{c}}^{+}$-baryon production in $\mathrm{pp}$, $\mathrm{p}$ $\mathrm{Pb}[20,21]$, and $\mathrm{Pb}-\mathrm{Pb}$ collisions [22] at the LHC might shed light on the hadronization mechanism for charm baryons. In particular, the $\Lambda_{\mathrm{c}}^{+} / \mathrm{D}^{0}$ yield ratio promises further insight into this process. In Fig. 6a this ratio, measured at mid rapidity as a function of $p_{\mathrm{T}}$ in pp collisions [20], is compared with expectations from perturbative QCD calculations and Monte Carlo event generators. None of the calculations reproduces the data, showing that charm fragmentation to baryons is not well understood. The situation is similar in $\mathrm{p}-\mathrm{Pb}$ collisions where a model which includes nuclear shadowing cannot describe the data over the full $p_{\mathrm{T}}$ range [20]. Similar conclusions have been drawn from measurements at forward rapidity [21]. $\Lambda_{\mathrm{c}}^{+}$baryon and $\mathrm{D}_{\mathrm{s}}^{+}$meson production have also been studied in $\mathrm{Pb}-\mathrm{Pb}$ collisions at $\sqrt{s_{\mathrm{NN}}}=5.02 \mathrm{TeV}$ [22]. Fig. 6b depicts the nuclear modification factor for these hadrons in comparison with $R_{\mathrm{AA}}$ of non-strange $\mathrm{D}$ mesons and charged particles. While the measured values for the heavy-flavor hadrons are consistent with each other within the combined statistical and systematic uncertainties a hint for a hierarchy $\left(R_{\mathrm{AA}}^{\mathrm{D}^{0}}<R_{\mathrm{AA}}^{\mathrm{D}_{\mathrm{s}}^{+}}<R_{\mathrm{AA}}^{\Lambda_{\mathrm{c}}^{+}}\right)$is visible, which might point to an important role of recombination mechanisms to charm hadronization in the dense medium. It is crucial to measure the yields of heavy-flavor baryons and strange heavyflavor mesons with precision because an enhancement of those can have a significant impact on the total heavy-flavor yields, which constitute a crucial baseline for the measurement of quarkonia in heavy-ion collisions. 
a)

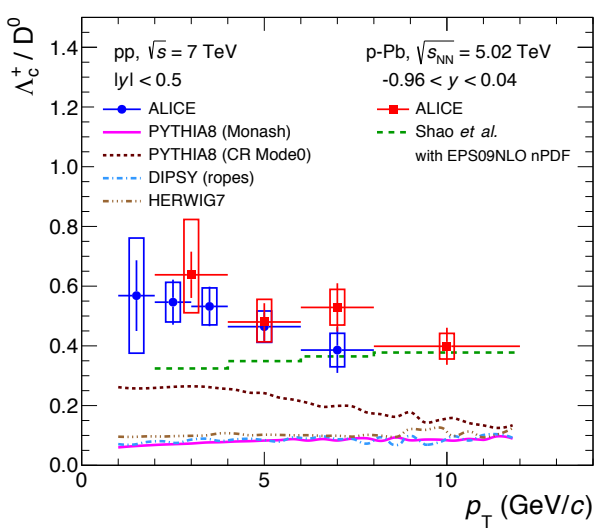

b)

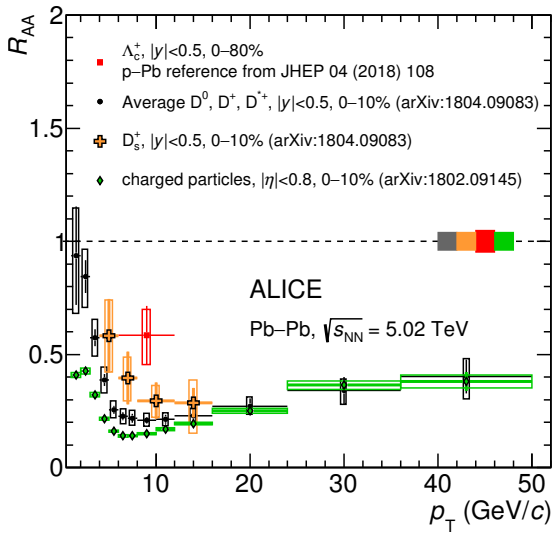

Figure 6: a) Yield ratio of $\Lambda_{\mathrm{c}}^{+}$baryons and $\mathrm{D}^{0}$ mesons in pp and $\mathrm{p}$-Pb collisions at $\sqrt{s_{\mathrm{NN}}}=7$ and $5.02 \mathrm{TeV}$, respectively, in comparison with model predictions (from [20]). b) $R_{\mathrm{AA}}$ of $\Lambda_{\mathrm{c}}^{+}, \mathrm{D}_{\mathrm{s}}^{+}$, and non-strange $\mathrm{D}$ mesons in $\mathrm{Pb}-\mathrm{Pb}$ collisions at $\sqrt{s_{\mathrm{NN}}}=5.02 \mathrm{TeV}$ (from [22]).

\section{Quarkonia}

Heavy charm and beauty quark-antiquark pairs can also form bound states, i.e. quarkonia. In a deconfined medium of sufficiently high energy density or temperature $T$ the quark-antiquark potential is screened by the surrounding partons, which is the QCD analogue of Debye screening [23]. The screening length $\lambda_{\mathrm{D}}$ depends on the temperature of the medium $\left(\lambda_{\mathrm{D}} \propto 1 / T\right)$ and the size of quarkonia depends on their binding energy $E_{\mathrm{b}}\left(r \propto 1 / E_{\mathrm{b}}\right)$. For $r>\lambda_{\mathrm{D}}$ a quarkonium state is not bound. Since different quarkonia have different binding energies the "sequential dissociation" and the resulting suppression pattern of quarkonium states was predicted to serve as a QGP thermometer [23]. When the density of heavy quarks in the deconfined system is large enough, which is the case for charm quarks at RHIC and, in particular, at the LHC, a competing production mechanism becomes important. Quarkonia can be (re)generated from deconfined heavy quarks and antiquarks, potentially during hadronization at the phase boundary [24].

Experimental evidence for $\mathrm{J} / \psi$ (re)generation is presented in Fig. 7a which shows the nuclear modification factor $R_{\mathrm{AA}}$ of $\mathrm{J} / \psi$ at forward rapidity as a function of the number of participants in $\mathrm{Pb}$ $\mathrm{Pb}$ collisions at $\sqrt{s_{\mathrm{NN}}}=2.76$ and $5.02 \mathrm{TeV}$ at the LHC and in Au-Au collisions at $\sqrt{s_{\mathrm{NN}}}=0.2 \mathrm{TeV}$ at RHIC [25] as well as $v_{2}$ of $\mathrm{J} / \psi$ in semi-central $\mathrm{Pb}-\mathrm{Pb}$ collisions at $\sqrt{s_{\mathrm{NN}}}=5.02 \mathrm{TeV}$ as a function of $p_{\mathrm{T}}$ at forward and at mid rapidity in comparison with various model predictions [26]. In central collisions $R_{\mathrm{AA}}$ grows with increasing $\sqrt{s_{\mathrm{NN}}}$ as does the density of charm quarks and the energy density in the deconfined QGP. This reduction of $\mathrm{J} / \psi$ suppression at larger $\sqrt{s_{\mathrm{NN}}}$ can only be explained by (re)generation being the dominant $\mathrm{J} / \psi$ production mechanism. This is confirmed by the observation of an enhancement of $\mathrm{J} / \psi$ yields at low $p_{\mathrm{T}}$ at mid rapidity, where the charm quark and energy density are even larger than at forward rapidity. Furthermore, if $J / \psi$ mesons are formed via (re)generation from deconfined charm quarks and antiquarks, which participate in the collective flow of the QGP (see Fig. $4 \mathrm{~b}$ ), also $\mathrm{J} / \psi$ mesons should pick up a positive flow. This is indeed the case as demonstrated in Fig. 7b. The measured positive $v_{2}$ [26] can only be reproduced in model calculations if $\mathrm{J} / \psi$ production via (re)generation is considered. The flow of primordial $\mathrm{J} / \psi$ produced from initial hard scattering processes is predicted to be much small than the $v_{2}$ observed. 
a)

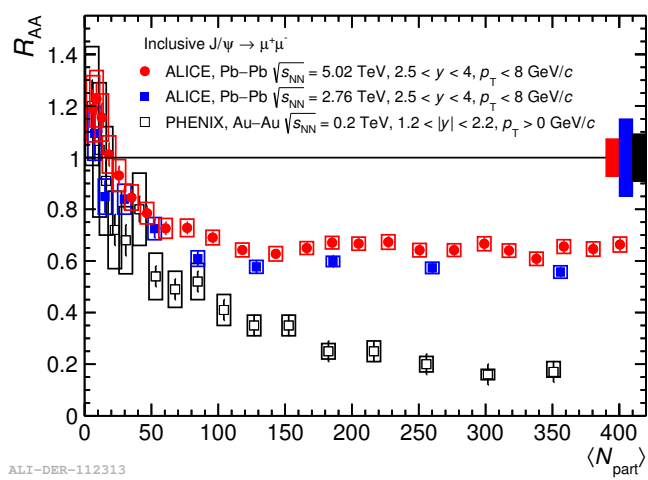

b)

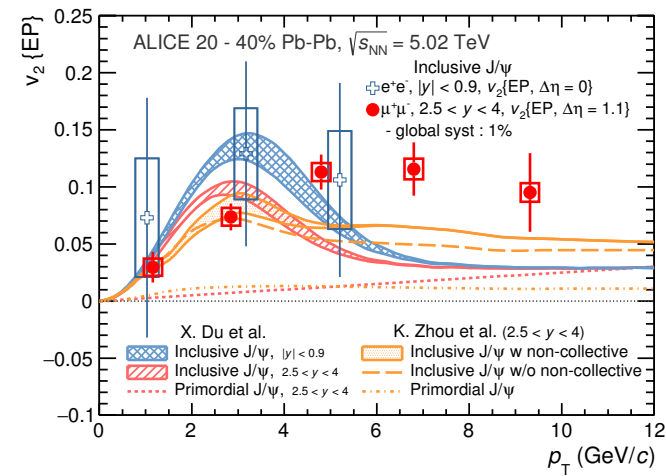

Figure 7: a) $R_{\mathrm{AA}}$ of $\mathrm{J} / \psi$ measured in the dimuon decay channel at forward rapidity as a function of the number of participants in Pb-Pb collisions at $\sqrt{s_{\mathrm{NN}}}=2.76$ and 5.02 TeV at the LHC and in Au-Au collisions at $\sqrt{s_{\mathrm{NN}}}=0.2 \mathrm{TeV}$ at RHIC (from [25]). b) Elliptic flow coefficient $v_{2}$ of $\mathrm{J} / \psi$ measured in semi-central $\mathrm{Pb}-\mathrm{Pb}$ collisions at $\sqrt{s_{\mathrm{NN}}}=5.02 \mathrm{TeV}$ as a function of $p_{\mathrm{T}}$ at forward and at mid rapidity in comparison with various model predictions (from [26]).

a)

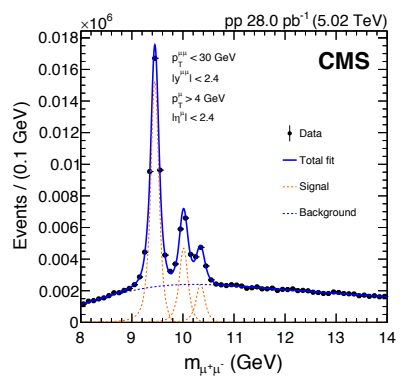

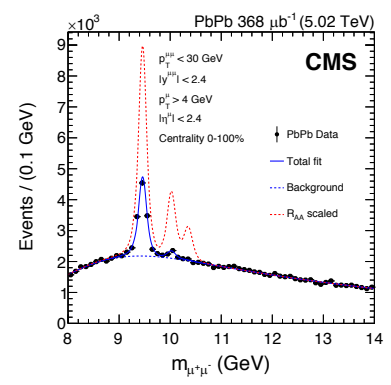

b)

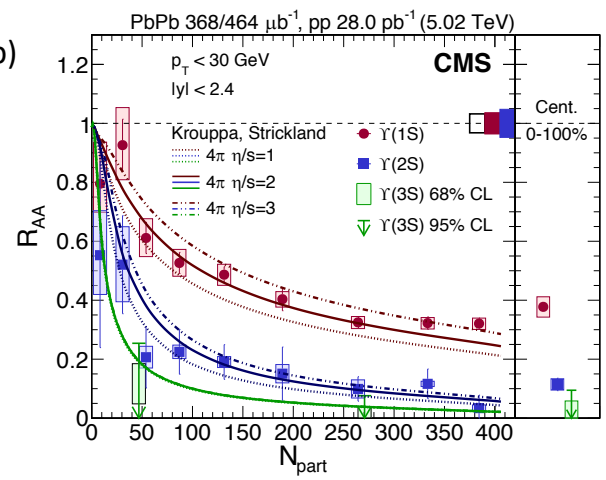

Figure 8: a) Dimuon invariant-mass distributions in $\mathrm{pp}$ (left) and $\mathrm{Pb}-\mathrm{Pb}$ collisions (right) at $5.02 \mathrm{TeV}$. The red line shows a fit of the $\Upsilon(1 \mathrm{~S}), \Upsilon(2 \mathrm{~S})$, and $\Upsilon(3 \mathrm{~S})$ signals in pp collisions and scaled with the number of binary collisions in $\mathrm{Pb}-\mathrm{Pb}$ collisions, respectively (from [27]). b) Corresponding $R_{\mathrm{AA}}$ for $\Upsilon(1 \mathrm{~S}), \Upsilon(2 \mathrm{~S})$, and $\Upsilon(3 \mathrm{~S})$ as a function of $N_{\text {part }}$ in comparison with model expectations (from [27]).

In the $\Upsilon$ sector the situation is distinctively different. In central $\mathrm{Pb}-\mathrm{Pb}$ collisions at top LHC energy about $100 c \bar{c}$ but only about $5 b \bar{b}$ pairs are produced and, therefore, (re)generation is not expected to be an important production mechanism of bottomonia. Instead, sequential suppression of $\Upsilon(1 \mathrm{~S}), \Upsilon(2 \mathrm{~S})$, and $\Upsilon(3 \mathrm{~S})$ yields seems to be at play in $\mathrm{Pb}-\mathrm{Pb}$ collisions at the LHC [27] as demonstrated in Fig. 8a. With increasing $\Upsilon$ mass and decreasing binding energy a more pronounced suppression of the $\Upsilon$ yields is observed. The centrality dependence of $R_{\mathrm{AA}}$ [27] is plotted for $\Upsilon(1 \mathrm{~S}), \Upsilon(2 \mathrm{~S})$, and $\Upsilon(3 \mathrm{~S})$ as a function of $N_{\text {part }}$ in Fig. 8b. A pattern reminiscent of the originally proposed sequential dissociation picture is observed. Models that implement color screening and (re)generation mechanisms during the dynamical evolution of the hot and dense medium allow to estimate the initial temperature of the QGP. Consistent values in the range of 629 to $641 \mathrm{MeV}$ [28] or 550 to $800 \mathrm{MeV}$ [29] are obtained, which place the medium produced in $\mathrm{Pb}-\mathrm{Pb}$ collisions at the LHC into the region of the QCD phase diagram which is governed by partonic degrees of freedom. 


\section{Conclusion}

The heavy-ion physics programs at the LHC and also at RHIC have delivered important new results concerning soft particle production and hard probes of the Quark-Gluon Plasma produced at ultra-relativistic collision energies. Correlations have been observed in $\mathrm{pp}$ and $\mathrm{p}-\mathrm{Pb}$ collisions which are similar to those in $\mathrm{Pb}-\mathrm{Pb}$ collisions. Also particle production evolves smoothly with charged-particle multiplicity in all systems, suggesting a common underlying mechanism. These observations have sparked intensive discussions and further experimental investigations as to whether a deconfined medium is also produced in high-multiplicity collisions of small systems. Data recorded in a six-hour run with Xe-Xe collisions at the LHC have provided a rich physics output. The comparison of nuclear modification factors in $\mathrm{Xe}-\mathrm{Xe}$ and $\mathrm{Pb}-\mathrm{Pb}$ collisions at similar charged-particle multiplicity should help to shed light on the path-length dependence of parton energy loss in the deconfined medium. The latter has also been addressed by jet and heavy-flavor hadron measurements. More precise heavy-flavor data have started to constrain the transport properties of the medium and address the charm hadronization mechanism in all systems. New data on charmonium production have shown evidence for (re)generation as dominant production mechanism and, consequently, have provided proof for deconfinement in heavy-ion collisions. The suppression pattern of $\Upsilon$ states has been shown to be consistent with sequential dissociation, thus serving as a thermometer for the Quark-Gluon Plasma.

\section{References}

[1] W. Busza, K. Rajagopal, W. van der Schee, Heavy-ion collisions: the big picture and the big questions, Annu. Rev. Nucl. Part. Sci. 68 (2018) 339, [arXiv: 1802.04801 ]

[2] F. Karsch, Lattice QCD at high temperature and density, Lect. Notes Phys. 583 (2002) 209, [hep-lat/0106019:]

[3] Y. Aoki, G. Endrodi, Z. Fodor et al., The order of the quantum chromodynamics transition predicted by the standard model of particle physics, Nature 443 (2006) 675, [hep-lat / 0611014 ]

[4] HotQCD Collaboration, The QCD crossover at zero and non-zero brayon density from Lattice QCD, submitted for publication in Nucl. Phys. A, [arXiv:1807.05607]

[5] ALICE Collaboration, Energy dependence and fluctuations of anisotropic flow in $\mathrm{Pb}$-Pb collisions at $\sqrt{s_{\mathrm{NN}}}=5.02$ and 2.76 TeV, JHEP 072018 103, [arXiv: 1804.0294 ] ]

[6] CMS Collaboration, Evidence for collectivity in pp collisions at the LHC, Phys. Lett. B 765 (2017) 193, [arXiv:1606.06198]

[7] ATLAS Collaboration, Measurement of long-range multiparticle azimuthal correlations with the subevent cumulant method in pp and $p+P b$ collisions with the ATLAS detector at the CERN Large Hadron Collider, Phys. Rev. C 97 (2018) 024904, [arXiv: 1708 . 03559]

[8] CMS Collaboration, Elliptic flow of charm and strange hadrons in high-multiplicity p-Pb collisions at $\sqrt{s_{\mathrm{NN}}}=8.16$ TeV, Phys. Rev. Lett. 121 (2018) 082301, [arXiv : 1804.09767 ]

[9] ALICE Collaboration, Enhanced production of multi-strange hadrons in high-multiplicity proton-proton collisions, Nature Physics 13 (2017) 535, [arXiv: 1606.07424 ]

[10] T. Sjöstrand, Collective effects: the viewpoint of HEP MC codes, submitted for publication in Nucl. Phys. A, [arXiv:1808.03117] 
[11] ALICE Collaboration, Transverse momentum spectra and nuclear modification factors of charged particles in Xe-Xe collisions at $\sqrt{s_{\mathrm{NN}}}=5.44 \mathrm{TeV}$, accepted for publication in Phys. Lett. B, [arXiv: 1805.04399]

[12] ALICE Collaboration, Transverse momentum spectra and nuclear modification factors of charged particles in $\mathrm{pp}, \mathrm{p}-\mathrm{Pb}$ and $\mathrm{Pb}-\mathrm{Pb}$ collisions at the LHC, accepted for publication in JHEP, [arXiv:1802.09145]

[13] CMS Collaboration, Charged-particle nuclear modification factors in $\mathrm{PbPb}$ and $\mathrm{pPb}$ collisions at $\sqrt{s_{\mathrm{NN}}}=5.02 \mathrm{TeV}$, JHEP 04 (2017) 039, [arXiv: 1611.01664$]$

[14] A. Kurkela, U. Wiedemann, B. Wu, Kinetic transport is needed to reliably extract shear viscosity from pA and AA data, arXiv: 1805.04081

[15] ATLAS Collaboration, Measurement of the nuclear modification factor for inclusive jets in $\mathrm{Pb}+\mathrm{Pb}$ collisions at $\sqrt{s_{\mathrm{NN}}}=5.02 \mathrm{TeV}$ with the ATLAS detector, submitted for publication in Phys. Lett. B, [arXiv: 1805.05635]

[16] ALICE Collaboration, Measurement of $D^{0}, D^{+}, D^{*+}$ and $D_{\mathrm{s}}^{+}$production in $\mathrm{Pb}-\mathrm{Pb}$ collisions at $\sqrt{s_{\mathrm{NN}}}=5.02 \mathrm{TeV}$, submitted for publication in JHEP, [arXiv : 1804.09083 ]

[17] R. Rapp et al., Extraction of heavy-flavor transport coefficients in QCD matter, Nucl. Phys. A 979 (2018) 21, [arXiv: 1803.03824$]$

[18] CMS Collaboration, Studies of beauty suppression via nonprompt $D^{0}$ mesons in PbPb collisions at $\sqrt{s_{\mathrm{NN}}}=5.02 \mathrm{TeV}$, submitted for publication in Phys. Rev. Lett, , [arXiv:1810.11102]

[19] Y.L. Dokshitzer, D. Kharzeev, Heavy quark colorimetry of QCD matter, Phys. Lett. B 519 (2001) 199, [hep-ph/0106202]

[20] ALICE Collaboration, $\Lambda_{c}^{+}$production in pp collisions at $\sqrt{s}=7 \mathrm{TeV}$ and in $p$-Pb collisions at $\sqrt{s_{\mathrm{NN}}}=5.02 \mathrm{TeV}, \mathrm{JHEP} 04$ (2018) 108, [arXiv : 1712.09581]

[21] LHCb Collaboration, Prompt $\Lambda_{c}^{+}$production in $p P b$ collisions at $\sqrt{s_{\mathrm{NN}}}=5.02 \mathrm{TeV}$, submitted for publication in JHEP, [arXiv:1809.01404]

[22] ALICE Collaboration, $\Lambda_{c}^{+}$production in Pb-Pb collisions at $\sqrt{s_{\mathrm{NN}}}=5.02 \mathrm{TeV}$, submitted for publication in Phys. Lett. B, [arXiv:1809.10922]

[23] T. Matsui, H. Satz, J/ $\psi$ suppression by quark-gluon plasma formation, Phys. Lett. B 718 (1986) 416

[24] P. Braun-Munzinger, J. Stachel, (Non)thermal aspects of charmonium production and a new look at J/ $\psi$ suppression, Phys. Lett. B 490 (2000) 196, [nucl-th/ 0007059 ]

[25] ALICE Collaboration, J/ $\psi$ suppression at forward rapidity in Pb-Pb collisions at $\sqrt{s_{\mathrm{NN}}}=5.02 \mathrm{TeV}$, Phys. Lett. B 766 (2017) 212, [arXiv:1606.08197]

[26] ALICE Collaboration, J/ $\psi$ elliptic flow in Pb-Pb collisions at $\sqrt{s_{\mathrm{NN}}}=5.02$ TeV, Phys. Rev. Lett. 119 (2017) 242301, [arXiv:1709.05260]

[27] CMS Collaboration, Measurement of nuclear modification factors of $\Upsilon(1 S), \Upsilon(2 S)$, and $\Upsilon(3 S)$ mesons in PbPb collisions at $\sqrt{s_{\mathrm{NN}}}=5.02 \mathrm{TeV}$, submitted for publication in Phys. Lett. B, [arXiv: 1805.09215]

[28] B. Krouppa, M. Strickland, Predictions for bottomonia suppression in $5.023 \mathrm{TeV} \mathrm{Pb-Pb} \mathrm{collisions,}$ Universe 2 (2016) 16, [arXiv: 1605 .03561]

[29] X. Du, M. He, R. Rapp, Color screening and regeneration of bottomonia in high-energy heavy-ion collisions, Phys. Rev. C 96 (2017) 054901, [arXiv: 1706.08670 ] 